\title{
Electromagnetic facilities and observing strategies for multi-messenger science: situation and future perspectives.
}

\section{Enrico Cappellaro*}

INAF, Osservatorio Astronomico di Padova, Padova (Italy)

E-mail: enrico.cappellaro@inaf.it

\begin{abstract}
The era of multi-messenger astrophysics began on 2017 Aug 17 with the discovery of a short GRB and of a kilonova associated with the GW170817 gravitational wave transient detected by the LIGO/Virgo interferometers. The observations confirm the predicted scenario for the merging of two neutron stars and demonstrated the potential of combining information derived from different messengers. We briefly summarise the challenges and opportunities for the multi-wavelength contribution to the forthcoming LIGO/Virgo runs of the Italian astrophysical community.
\end{abstract}

GRAvitational-waves Science\&technology Symposium - GRASS2018

1-2 March 2018

Palazzo Moroni, Padova (Italy)

\footnotetext{
* on behalf of the GRAWITA collaboration (http://grawita.inaf.it
} 


\section{Discovery of a kilonova}

On 17 August 2017, the LIGO/Virgo collaboration announced the discovery of the GW signal from the coalescence of two neutron stars (GW170817) registered only $1.8 \mathrm{sec}$ before a short GRB detected by the FERMI GMB instrument and located in a (partially) overlapping sky box [1]. The small sky box (few tens degrees), the short distance $(40 \mathrm{Mpc})$ and the prediction that the binary neutron star (BNS) merging ejects enough material to produce a bright optical transient motivated a world-wide search involving over eighty observing facilities [2]. Knowing where and what to search, hemisphere), a 17 mag transient was discovered (AT2017gfo) soon after sunset in Chile (the target was located in the southern. In fact, in the elapsed time between the first observation, $10.86 \mathrm{~h}$ after the GW trigger, and the discovery announcement by the SWOPE team [3], $1.5 \mathrm{~h}$ later, six telescopes secured images of the same source[2]. The transient luminosity rapidly declined (faster in blue bands) and in less than $10 \mathrm{~d}$ it dropped below the detection threshold for small size telescopes [4].

One interesting question to address is whether the optical transient would be found if there was no GW (or GRB) trigger. Nowadays there are many sky surveys with the proper cadence to detect fast transients similar to AT2017gfo. For instance, the DLT40 supernova search [5] since a couple of years is monitoring with daily cadence (weather conditions permitting) a sample of two thousand bright galaxies closer than 40 Mpc. NGC 4993, the host galaxy of AT2017gfo, is in the DLT40 target list but in August last year the monitoring campaign of this galaxy was suspended due to the Sun angular distance constraint. Therefore, though DLT40 had an adequate observing strategy, AT2017gfo would not be discovered without the GW trigger. The same conclusions applies to other similar transient surveys, i.e. LSQ, ATLAS, SkyMapper, ASAS-SN and Catalina ([6] and references therein). Indeed, considering the low rate of BNS, the expected discovery rate of AT2017gfo-like events in the DLT40 survey is $1 / 20 \mathrm{yr}^{-1}$ [7]. For a detailed discussion of the optical search strategies, problems and prospects see $[8,9]$.

Soon after the discovery, a spectrum was taken to verify the nature of the optical transient [10]. This early spectrum was almost featureless and fairly blue $\left(T_{\mathrm{BB}}=11000^{\circ} \mathrm{K}\right)$, i.e. similar to that of early core collapse SNe [2]. No other transient was found in the small search volume and the follow up could be focused on AT2017gfo only. In this activity a major role was played by the European Southern Observatory (ESO) with its suite of telescopes/instruments. The key instrument turned out to be the X-Shooter spectrograph mounted at the VLT, which provided daily monitoring of the whole optical/near-infrared spectral range with excellent resolution and signal-to-noise ratio $[11,12]$. These observations show that the spectrum change very rapidly: in two/three days the spectral energy distribution shifts from visual to near-infrared (NIR) and the spectrum becomes unlike that of any known SN type (Fig. 1). Instead, the spectral features well match those predicted by models for the ejecta of BNS mergers [13]. In these models, explosive nucleosynthesis in material with high neutron fraction proceeds through rapid neutron capture (r-process) resulting in a high abundance of heavy elements [14]. Among others there are elements with very high opacity (in particular lanthanides) that dominate the radiation transport and make for an infrared transient.

Actually, a detailed comparison of models with observations shows evidences of multiple components with different masses, expansion velocities and lanthanide abundances [11]. In particular, the presence of a blue component suggests that a hot neutron star survives at least for tens of 
milliseconds after the merging. When this occurs, neutrino irradiation lowers the neutron fraction and part of the ejecta can have very low lanthanide abundance [15, 14]. We stress that the presence of multiple components in the kilonova ejecta conflicts with the basic assumptions of current models, which are based on spherical symmetry, local thermodynamic equilibrium, and uniform abundances. When comparing theory with observations we have also to consider the large uncertainties in the current atomic line lists for lanthanides and other relevant heavy element species (eg. actinides).

Despite the uncertainties, the presence of r-process elements in the ejecta appears undisputed. On this basis and assuming the predicted r-process abundance ratio, we can infer the presence in the ejecta of roughly $100 \mathrm{M}_{\text {Earth }}$ of gold.

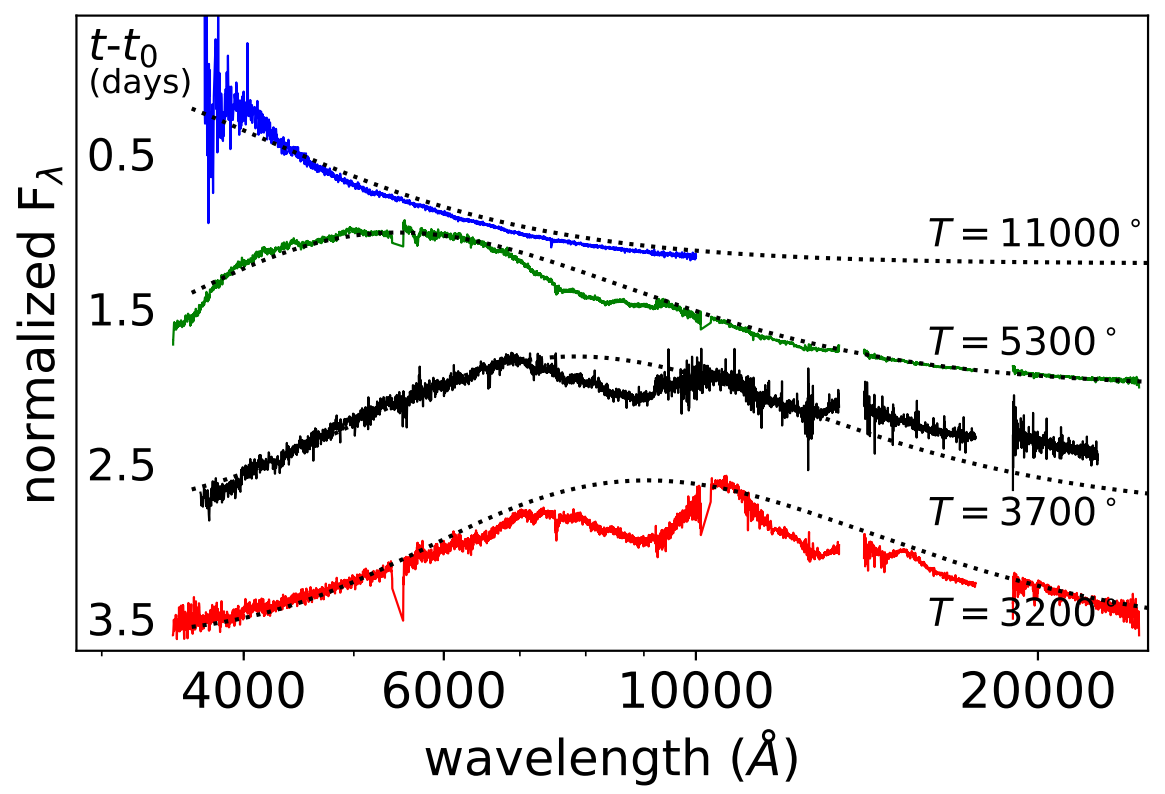

Figure 1: Spectra of the kilonova AT2017gfo in the first few days are compared with the spectral energy distribution of black-bodies with different temperatures. References of the observed spectra are as follows: $0.5 \mathrm{~d}$ [10] (blue line), 1.5 and 3.5d [11] (green and red lines), 2.5d [12] (black line). All spectra, but the first one, have been obtained with the X-Shooter spectrograph at ESO-VLT.

Following the optical transient identification, $9 \mathrm{~d}$ after the trigger the source was detected in $\mathrm{X}$ ray [16] and soon after $(+16 \mathrm{~d})$ it was detected at radio frequencies [17, 18]. This delayed radiation along with the properties of the faint sGRB observed soon after merging, were attributed to a beamed jet seen at a moderate viewing angle $(20-40 \mathrm{deg})$. Alternatively, this late emission may originate from a broadly collimated, mildly relativistic cocoon [19]. The target was observed again three months later when the object re-emerged out of the Sun glow and the non-thermal emission was found still on the rising. With the available observations it is not yet possible to choose among the two alternative scenarios [20] and monitoring of the source for a few years may be required to settle the issue. 


\section{Preparing for the future}

GW170817 is to date the only GW event with an associated electromagnetic source and is therefore the reference for planning the follow up of the forthcoming LIGO/VIRGO runs. Yet, we should be prepared for significant diversity between BNS merger outcomes depending on the system physical parameters, eg. binary component masses, or on the specific observing conditions, eg. viewing angles. In fact, diversity will likely be a key for understanding the physics of BNS mergers. In this context the on-going efforts to improve the sensitivity of GW interferometers are instrumental. In the next run (O3), LIGO is expected to reach $120 \mathrm{Mpc}$ for BNS mergers almost doubling the previous limit and the predicted rate of GW triggers scales up to $1-30 \mathrm{yr}^{-1}$ for BNS and $10-100 \mathrm{yr}^{-1}$ for BBH mergers respectively [21].

Yet, most targets will be at larger distance than GW170817. This implies that the prompt sGRB emission may be missed but in the very unlikely case that the viewing angle is aligned with the jet. On the other hand, the optical kilonova from a BNS merger occurring at the limit distance of 120 Mpc would have an apparent magnitude at peak $\sim 20$, which is still detectable with small/medium size telescopes. Thus, if the sky box is not too large, finding the optical transient is feasible but with two complications: i) in the large volume, the contamination from other variable sources will be large and there will be the need to scrutinise a large number of candidates; ii) achieving a good signal-to-noise ratio for the critical spectroscopic observations of these candidates will require large telescopes. In this contest, the GRAWITA Italian collaboration was among the promoters of a European wide initiative (ENGRAVE: Electromagnetic counterparts of gravitational wave sources at the Very Large Telescope) aimed to assure an optimal use of the most valuable ESO observing facilities,

At the same time Italian researcheres are preparing for the use of the National facilities that, although in less favourable sites, allow for great flexibility, eg. the Schmidt telescopes in Campo Imperatore and Asiago for the transient search or the $1.8 \mathrm{~m}$ telescope in Asiago and the $1.5 \mathrm{~m}$ telescope in Loiano for the spectroscopic follow up.

In the long time scale, the improvement in the sensitivity of the interferometers will demand for fainter limits for the electromagnetic follow up. In this respect, two instruments are expected to give a major contribution to the multi-messenger science case in the next few years: i) the Large Synoptic Survey Telescope (LSST) is a $8.4 \mathrm{~m}$ telescope with a large field of view (10 sq.deg.) that will monitor the full accessible sky every few nights (https://www.lsst.org). Expected to enter in operation in 2023, LSST will allow to search for very faint optical counterparts even in moderately large sky box. ii) the James Webb Space Telescope (JWST) is a $6.5 \mathrm{~m}$ space telescope that will be launched in 2020 (https://www.jwst.nasa.gov) and will reach unprecedented sensitivity in the near-mid infrared both for imaging and spectroscopy.

INAF is committed to contribute to the development of three major facilities of the next decade, namely: i) the ESO Extremely Large Telescope (ELT) that with its 39m mirror will be the world largest optical telescope (https://www.eso.org/sci/facilities/eelt/). Along with the mirror size, the crucial ingredient for the extreme performances of ELT is the advanced adaptive optics technology. In this project, INAF major contribution is a module for multi-conjugate adoptive optics (MAORY, http://wwwmaory.oabo.inaf.it); ii) the Cherenkov Telescope Array (CTA) is a facility with tens of telescopes distributes in two continents aimed to detectCherenkov radiation from very high energy 
photons and cosmic rays (https://www.cta-observatory.org); iii) the Square Kilometer Array (SKA) will feature hundreds of antennas in Australia and South Africa for the deep survey of the radio sky (https://www.skatelescope.org).

INAF is also contributing to prepare the future of high-energy space facilities with two projects: i) Theseus a proposed ESA medium-size mission (M5) designed for the study of high energy transients (https://www.isdc.unige.ch/theseus/, [22]) and ii) the Athena (http://www.the-athena-x-rayobservatory.eu) X-ray observatory is a class L (Large) mission included in the ESA Cosmic Vision 2015-2025 programme.

It should be stressed that because of the expected large number of triggers an efficient search strategy requires a network of dedicated, flexible small scale facilities to distill the candidates and identify the targets for highly over-subscribed facilities. While for imaging a fair number of small robotic telescopes are already available, a network of medium size telescope with spectroscopic capability is not available. Yet, it is clear that spectroscopic classification of transient candidates will become a bottleneck and it is then crucial that dedicated instruments are developed for this task. With this motivations, INAF is leading the design, building and operation of SOXS, an optical/NIR spectrograph to be installed at the ESO-NTT, which will be devoted mainly to transient science.[23]

Along with new instruments, we also need to cooperate for a change of attitude of the astronomical community, devoting more efforts to establish a world-wide coordination in contrast to the current fierce competition. The above mentioned European ENGRAVE collaboration is a first step in this direction but further initiatives will be necessary.

The new era of multi-messenger astrophysics has started and Italian researches are ready to play a key role in exploiting the new generation of multi-wavelength facilities.

\section{References}

[1] Abbott, B. P., Abbott, R., Abbott, T. D., et al., 2017, ApJL, 848, L13

[2] Abbott, B. P., Abbott, R., Abbott, T. D., et al., 2017, ApJL, 848, L12

[3] Coulter, D. A., Foley, R. J., Kilpatrick, C. D., et al., 2017, Science, 358, 1556

[4] Villar, V. A., Guillochon, J., Berger, E., et al., 2017, ApJL, 851, L21

[5] Valenti, S., Sand, D. J., Yang, S., et al., 2017, ApJL, 848, L24

[6] Drake A. J., Djorgovski S. G., Mahabal A. A., et al., 2017, The Astronomer's Telegram, 10856, 1

[7] Yang, S., Valenti, S., Cappellaro, E., et al., 2017, ApJ, 851, L48.

[8] Yang, S., 2018, in proceeding of GRASS2018, PoS(GRASS2018)037

[9] Grado, A., 2018, in proceeding of GRASS2018, PoS(GRASS2018)025

[10] Shappee, B. J., Simon, J. D., Drout, M. R., et al., 2017, Science, 358, 1574

[11] Pian, E., D'Avanzo, P., Benetti, S., et al., 2017, Nat, 551, 67

[12] Smartt, S. J., Chen, T.-W., Jerkstrand, A., et al., 2017, Nat, 551, 75

[13] Kasen, D., Badnell, N. R., \& Barnes, J., 2013, ApJ, 774, 25

[14] Tanaka, M., Utsumi, Y., Mazzali, P. A., et al., 2017, PASJ, 69, 102 
[15] Kasen, D., Metzger, B., Barnes, J., et al., 2017, Nat, 551, 80

[16] Troja, E., Piro, L., van Eerten, H., et al., 2017, Nat, 551, 71

[17] Alexander, K. D., Berger, E., Fong, W., et al., 2017, ApJL, 848, L21

[18] Hallinan, G., Corsi, A., Mooley, K. P., et al., 2017, Science, 358, 1579

[19] Lazzati D., López-Cámara D., Cantiello M., et al., 2017, ApJ, 848, L6

[20] Margutti, R., Alexander, K. D., Xie, X., et al., 2018, ApJL, 856, L18

[21] Dent, T., Pankow, C., 2018, LIGO-DCC G1800370

[22] Amati, L., O’Brien, P., Goetz, D., et al., 2017, ArXiv e-prints , arXiv:1710.04638.

[23] Schipani, P., Claudi, R., Campana, S., et al. 2016, Proc. SPIE, 9908, 990841 